The How's and Why's of Face Processing:

Level of Construal Influences the Holistic Processing of Human Faces

Natalie A. Wyer, Timothy J. Hollins, \& Sabine Pahl

University of Plymouth

Word Count: 3034

This work was supported by a grant from the Economic and Social Research Council (ES/G035563/1). The authors wish to thank Chris Longmore for his feedback on this work and Mark Cooper for his programming help.

Correspondence regarding this manuscript, including requests for stimulus materials and raw data, may be addressed to the first author by post at: School of Psychology, University of Plymouth, Drake Circus, Plymouth PL4 8AA, United Kingdom; by telephone at +44 1752 584869; or by email at natalie.wyer@plymouth.ac.uk. 
Face recognition and identification are optimised by holistic processing. Various visualspatial manipulations appear to have transfer effects on holistic face processing. The present experiment tests the effects of a semantic manipulation - of construal level - on holistic processing as measured by composite congruency effects. Participants completed two blocks of trials. The first served as a baseline, whilst the second included a manipulation of construal level. High-level construal resulted in stronger congruency effects, indicative of greater holistic processing (relative to baseline and to low-level construal). These results have implications for conceptualisations of both construal level and holistic processing. 
The term 'holistic' has been applied to a range of psychological processes, from human face perception to action identification. In the face processing literature, research shows that holistic processing (i.e., attending to a face as a whole rather than to its constituent features) is advantageous for identifying to whom a face belongs or recognizing whether it has been seen before (Yin, 1969; Young, Hellawell, \& Hay, 1987). In the social cognition literature, researchers have investigated the causes and consequences of construing actions in a relatively specific/concrete vs. holistic/abstract manner (Trope \& Liberman, 2010; Vallacher \& Wegner, 1989). However, there have been few attempts to determine whether holistic face processing is associated with (or influenced by) holistic processing of semantic information. The current research examines this question by testing the impact of construal level (Trope \& Liberman, 2010) - that is, whether actions are construed in an abstract or concrete manner - on holistic face processing.

There are precedents for speculating that processing manipulations in one context might transfer to face processing tasks. To date, such demonstrations have involved primarily visual-spatial processing manipulations. For example, the prototypical stimulus for such research is the Navon letter (Navon, 1977), which is a larger letter shape made up from smaller letter features. (e.g. a large T made up of small h's). Using such stimuli, attention may be oriented to the global or local level, and such orientation towards either global or local levels causes transfer effects across items.

Transfer effects have also been demonstrated from processing of Navon stimuli to subsequent performance on a face identification tasks. Numerous studies have tested the ability of eyewitnesses to identify someone (encountered earlier) in a line-up, contingent upon an orientation task just prior to test. Compared to control conditions, participants who are oriented towards the 'global' shape of Navon letters are later more accurate at identifying the perpetrator, whilst those oriented towards the 'local' features are less so (Macrae \& Lewis, 
2002; Perfect, 2003; Perfect, Weston, Dennis, \& Snell, 2008). Thus, the transfer from the Navon task to the identification task is not a property of the stimuli (i.e., the target or the lineup as a whole), but is the result of the orientation toward them. Importantly, Gao, Flevaris, Robertson and Bentin (2010) showed that prior orientation to the global features of Navon stimuli led to an increased incongruency cost in a composite study, suggesting that global orientation promotes a holistic processing style.

Transfer effects also emerge from another eyewitness memory paradigm, the verbal overshadowing effect (VOE; Schooler \& Engstler-Schooler, 1990). VOE is the negative effect of verbal description on subsequent recognition of a face in a line-up. Crucially, this effect occurs even following a description of a different face (Dodson, Johnson \& Schooler, 1997) or an inanimate object (Westerman \& Larsen, 1997), and so cannot be attributed to a failure of source memory. Instead, it suggests that the tendency to describe objects or faces in terms of their features promotes a shift in processing (from holistic to feature-based) which later impairs face recognition (Schooler, 2002).

Such research suggests that verbal processing manipulations can alter visual processing of faces. Yet, even in the verbal overshadowing paradigm, participants' verbal descriptions focus on a visual stimulus. This raises the question of whether processing manipulations that are completely independent of visual stimuli might also influence face processing in general and holistic processing in particular. One manipulation that has received considerable attention in the social cognition literature is construal level. Construal Level (CL) theory posits that any action can be identified in more general/abstract or more specific/detailed ways (Trope \& Liberman, 2010). For example, driving a car can be identified as making progress towards a destination (high-level construal) or as using the steering wheel, accelerator, and clutch (low-level construal). Research on CL theory has 
established that construal level manipulations impact a wide range of social, cognitive, and perceptual tasks.

Construal level has also been linked to social memory, including memory for faces. Wyer, Perfect, and Pahl (2010) found that manipulations of temporal distance (a proxy for construal level) influenced performance on a face recognition task. Participants who considered an event in the near future (requiring low-level construal; Trope \& Liberman, 2003) were less accurate in identifying a confederate (encountered earlier) than were those who considered the same event in the distant future (encouraging high-level construal). Face recognition accuracy was correlated with measures of construal (e.g., self-reports of thinking in abstract vs. detailed terms, use of inclusive vs. discrete categories). This suggests that highlevel construal induces holistic processing and by extension facilitates face recognition (see also Hunt \& Carroll, 2008).

Yet, correlations of construal with face recognition accuracy are only suggestive of a link between construal level and holistic processing, because the underlying processes leading to accurate recognition are unknown (indeed, temporal distance manipulations may influence construal and face recognition via different mechanisms). Notably, the data do not rule out the possibility that construal level changed motivation towards the identification task. More compelling would be evidence that construal level had a differential effect on face processing under conditions where access to holistic information was either intact or disrupted. The present experiment was designed to provide such a test. We adopted the congruency paradigm to determine whether construal level influences congruency effects within the composite task.

\section{The Current Experiment}

The composite face effect is the well-established finding that face halves are more difficult to identify or recognize when presented with the remainder of a different face. For 
example, whilst it may be easy to identify the top half of George Clooney's face under normal circumstances, when the bottom half is replaced by someone else's face (e.g., George Bush), identification becomes much more difficult. The decline in face identification or recognition performance for such incongruent composites is argued to be a direct measure of holistic face processing (Richler, Cheung, \& Gauthier, 2011; Richler, Palmeri, \& Gauthier, 2012).

Various versions of the composite effect have been developed. A recent variation, adopted for the current study, was developed to control for response-bias shifts by independently manipulating the congruence of the response associated with the top and bottom half-face and the match between the study and test half-faces (e.g. Richler, Gauthier, Wenger, \& Palmeri, 2008; Richler et al, 2011). Congruent trials are those on which responses to the top and bottom of the test composite are the same (i.e., both halves are the same as the study face, or both halves are different) whilst incongruent trials are those on which responses to the top and bottom of the test composite are different (i.e., one half is the same as, but the other half different from, the study face).

The present experiment used a congruency paradigm to test for construal effects on holistic processing. Recent research indicates that the composite face effect is sensitive to manipulations that are believed to influence holistic processing. For example, Gao et al. (2011) reported that a global (vs. local) version of a Navon letter matching task resulted in greater disruption for incongruent composites. Similarly, Curby, Johnson, and Tyson (2012) demonstrated that positive mood (believed to promote holistic processing) also led to increased congruency effects. Importantly, a key aspect of this paradigm is that holistic processing leads to impairments in performance. Thus, if the effects of construal on face recognition in previous experiments (e.g., Hunt \& Carroll, 2008; Wyer et al, 2010) were due to holistic processing we would predict a stronger congruency effect, shown by worse 
performance in the high level construal condition. In contrast, if previous effects were due to a general factor like motivation, we would expect high-level construal to lead to superior performance.

We adopted a combination of Gao et al's (2011) and Curby et al's (2012) designs. Participants completed two blocks of trials. The first block consisted of a series of composite trials which required participants to judge whether or not the top half of a test facial composite matched that of a study composite. The second block included trials of the same sort, but intermittently required participants to answer questions about how or why they engaged in various actions. Thus, we obtained an estimate of each participant's baseline congruency effect in Block 1, against which we could assess changes as a function of our construal manipulation in Block 2. We expected that participants in the high-level construal condition - but not those in the low-level construal condition - would show increased congruency effects in Block 2 relative to Block 1.

Method

\section{Participants}

119 participants $\left(62\right.$ female, $\left.M_{\text {age }}=38.5\right)$ were recruited via the online crowdsourcing service 'Crowdflower' and completed the study in exchange for $\$ 4.00$. Of these, 12 participants terminated the experiment following Block 1 and were therefore excluded from analyses, yielding a final sample of 107 participants. Sample size was determined using GPower, which indicated a required sample of 34 to detect a medium-sized interaction (construal X congruency) effect $(f=.25)$ with $80 \%$ power, or a sample of 54 to achieve $95 \%$ power. Because this experiment was conducted online (and we anticipated greater 'noise'), we obtained a larger sample. GPower estimated that we achieved $99 \%$ power with our actual sample size (53-54 per between-participants condition).

Design 
The experiment utilised a 2 X 2 X 2 mixed design, with Construal Level (high vs. low) manipulated between-participants, and Block (1 vs. 2) and Congruence (congruent vs. incongruent) varying within-participants.

\section{Materials}

Two sets of 160 composite faces (or 80 study-test pairs) were prepared. Composite faces were formed using faces (converted to greyscale) from the Glasgow Face Database (Burton, White \& McNeill, 2010), cropping each of two faces across the bridge of the nose before combining the top of one face with the bottom of the same or a different face. Following Richler \& Gauthier (2014), a full design was used to avoid confounds due to response bias. In our version of the task, study composites were formed by re-combining the top and bottom halves of the same face. Four types of test composites were constructed. Congruent trials included 20 test composites that matched both the top and bottom of the study composite and 20 test composites that were formed by re-combining the top and bottom of a different (same-sex) face. Incongruent trials included 20 test composites with the same top but different bottom than the study composite and 20 test composites with the same bottom but different top than the study composite (see Figure 1).

\section{Procedure}

The experiment involved two blocks of 80 trials, each of which included an entirely different set of composites. Each composite set (A and B) was presented in Block 1 for half of the participants and in Block 2 for the other half. Each trial comprised a red fixation cross (500ms), a study composite face $(250 \mathrm{~ms})$, a blank screen $(250 \mathrm{~ms})$, and finally a test face (2000ms or until a response was registered, whichever occurred first). The inter-trial interval was $800 \mathrm{~ms}$. The on-line experiment was programmed in Java to pre-load the entire experiment when a participant accessed it; thus, variability in the speed of the internet connection should not have affected the precision of stimulus presentation. 
Before starting the experiment, participants were instructed to determine whether the top half of the test face was the same or different than the top half of the study face, and to respond using one of two keys on the keyboard. A reminder of which keys to use appeared beneath each test face. Before beginning the experiment proper, participants were given 4 practice trials (using composites formed of characters from The Simpsons) which they were able to repeat if they wished.

After the practice trials, participants completed Block 1. This allowed us to derive a baseline level of the congruence effect for each participant, prior to introducing the Construal Level manipulation. Next, participants were given instructions for Block 2, which varied according to their Construal Level condition. Participants in the high-level condition were instructed that, prior to each set of 4 study-test trials, they would be asked to provide brief answers to questions about why they do a variety of actions (e.g., 'Why do you brush your teeth?'). Participants in the low-level condition were instructed that they would be answering questions about how they do the same actions (e.g., 'How do you brush your teeth?'). ${ }^{1}$ Participants were given an example question and answer matched to their condition. Participants were given up to 10 seconds to type their response. They then completed the composite trials that followed in the same manner as in Block 1.

\section{Results}

D-prime scores $^{2}$ were computed separately for congruent and incongruent trials in Block 1 and Block 2, and were analysed using a three-way mixed model ANOVA in which Block (1 vs. 2) and congruence (congruent vs. incongruent) were entered as repeatedmeasures factors and construal level (high-level vs. low-level) was a between-participants

\footnotetext{
${ }^{1}$ Task difficulty did not vary between conditions; we confirmed that there were no betweencondition differences in the number of non-responses (e.g., trials where the response deadline passed without a response being generated).

${ }^{2}$ In calculating $d$-prime, hit rates of 0 were replaced by $1 / n$ and hit rates of 1 were replaced by $(\mathrm{n}-1) / \mathrm{n}$.
} 
factor. Preliminary analyses that included the counterbalancing factor of picture set indicated that this had no effect, and thus the results reported here exclude that factor.

Replicating prior research (Young et al, 1987; Richler et al, 2011, 2012), analyses of d-prime scores yielded a significant main effect of congruence, $F(1,105)=428.36, p<.001$, $\eta_{p}{ }^{2}=.80$ such that performance was superior for congruent $(\mathrm{M}=2.30, \mathrm{SE}=.08)$ than for incongruent trials $(\mathrm{M}=0.39, \mathrm{SE}=.07)$. This effect was moderated by a significant two-way interaction with Block, $F(1,105)=8.99, p=.003, \eta_{p}^{2}=.08$. Critically, this was further moderated by the predicted significant three-way (Congruence X Construal Level X Block) interaction, $F(1,105)=8.48, p=.004, \eta_{p}^{2}=.08($ see Figure 2$)$.

To decompose the significant three-way interaction, the Congruence X Construal Level interaction was analysed separately in Block 1 and Block 2. As expected, there was only a significant effect of Congruence in Block $1, F(1,105)=312.46, p<.001, \eta_{p}{ }^{2}=.75$, which was not moderated by Construal Level (the manipulation of which had not yet been introduced $), F(1,105)<1$. However, in Block 2, the significant effect of Congruence $(F(1$, $\left.105)=307.05, p<.001, \eta_{p}^{2}=.75\right)$ was qualified by a significant interaction with Construal Level, $F(1,105)=6.51, p=.012, \eta_{p}^{2}=.06$. Simple effects analyses indicated that High-Level Construal impaired performance on Incongruent trials $(M=0.09, S E=.12)$ compared to Low-Level Construal $(M=0.50, S E=.12), F(1,105)=5.64, p=.019, \eta_{p}{ }^{2}=.05$. In contrast, Construal Level had no notable effect on responses to Congruent trials $\left(M_{\text {High-Level }}=2.47, S E\right.$ $\left.=.13 ; M_{\text {Low-Level }}=2.28, S E=.13\right), F(1,105)=1.16, p=.28, \eta_{p}^{2}=.01$.

\section{Discussion}

The present experiment provides a critical test of the effect of construal level on the holistic processing of faces. Participants exposed to questions about 'why' events occur (our high-level construal manipulation) produced a significantly larger congruency effect on a composite face matching task than did those exposed to questions about 'how' the same 
events occur (our low-level construal manipulation). Notably, whilst the congruency effect increased from Block 1 to Block for high-level participants 2, there was little change for lowlevel participants. This finding suggests that high-level construal increased holistic processing relative to baseline levels, whereas low-level construal had little effect. The experiment reported here thus provides direct evidence that a semantic (and non-visual) construal task taken from the social cognition literature affects the holistic processing of faces. These findings have at important implications for our understanding of both construal level and holistic face processing.

The present study is the first to provide direct evidence that a manipulation that induces holistic processing using a completely non-visual-spatial, semantic task results in differences in holistic face processing. Although studies reported by Wyer et al (2010; see also Hunt \& Carroll, 2008) indicated that construal level altered face recognition performance, they did not identify the processes involved. In the current work, we demonstrated that highlevel construal promotes holistic processing, leading to a disadvantage when it comes to matching incongruent (but not congruent) facial composites. Thus, like the experimental work on mood effects reported by Curby et al (2012), non-visual and visual processing styles appear to be linked such that manipulations of one have an impact on the other.

Through what mechanism might construal level alter the way that faces are processed? One possibility is that construal level manipulations induce attentional biases that are generalised to subsequent processing tasks. For example, tasks that induce high-level construal may bias perceivers to attend to holistic information in general to the neglect of detailed information (Trope \& Liberman, 2010). When subsequently asked to match composite faces, they may apply this holistic processing bias to those faces. In contrast, tasks that induce low-level construal may bias attention towards other aspects of information presented, but leaves holistic processing unchanged. 


\section{Summary and Conclusions}

The present work establishes a novel link between semantic processing (i.e., construal level) and visual (face) processing. Further research is needed to specify what aspect of judgment (e.g., perception of the test stimulus, retrieval of the study stimulus, or decision about their similarity) is altered by construal, and what underlying mechanism links construal level to visual processing style. However, the experiment reported here represents an important step towards determining whether a common mechanism underlies holistic processing of both visual and semantic information. 


\section{Figure Captions}

Figure 1 (a) Trial Structure. Block 1 consisted of 40 trials presented in random order. Block 2 consisted of an additional 40 trials in random order, with a one-item construal manipulation presented before every 4 trials. Letters (A, B, C, D) shown within faces indicate distinct face halves. (b) Construal manipulation. Trial sequence in Block 2.

Figure 2. Accuracy (average d' with standard errors) for congruent vs. incongruent composites in Block 1 (prior to manipulation) and Block 2 (with manipulation) as a function of construal level. 
Fixation $(500 \mathrm{~ms})$

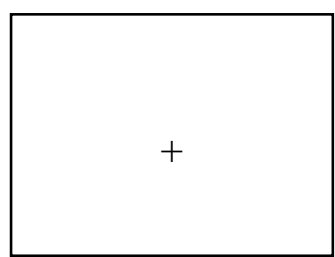

Study $(250 \mathrm{~ms})$

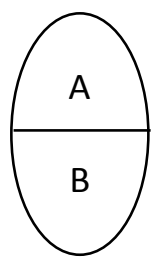

Blank (250ms)

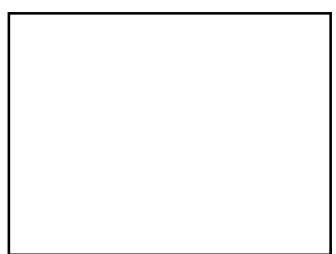

Test $(<2000 \mathrm{~ms})$ - one of 4 composite types
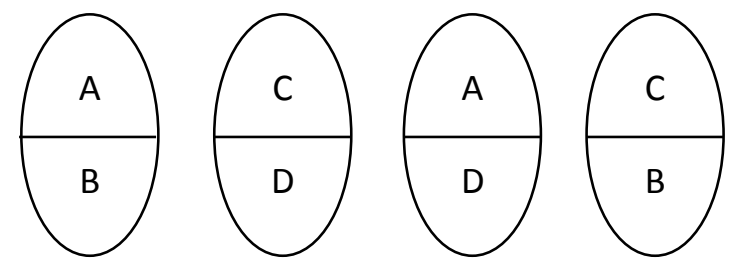

Correct Response: Congruency:

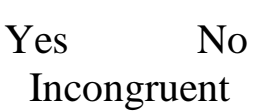

(b)

Construal Prompt with response box (Response deadline: 10 seconds)

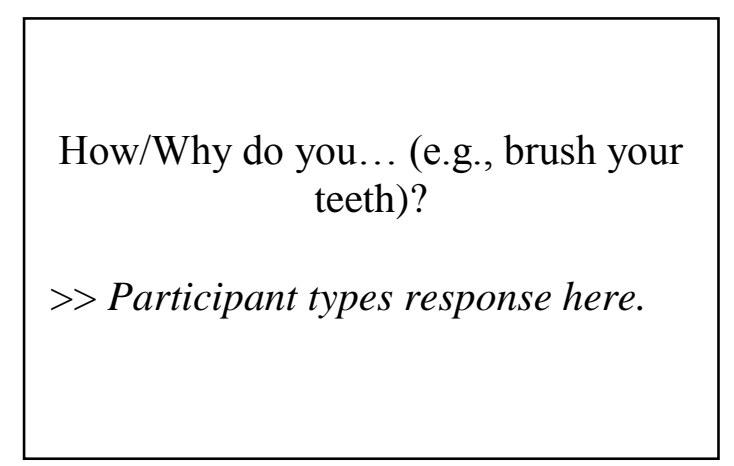

Four trials (random selected from set) as described in Fig. 1a
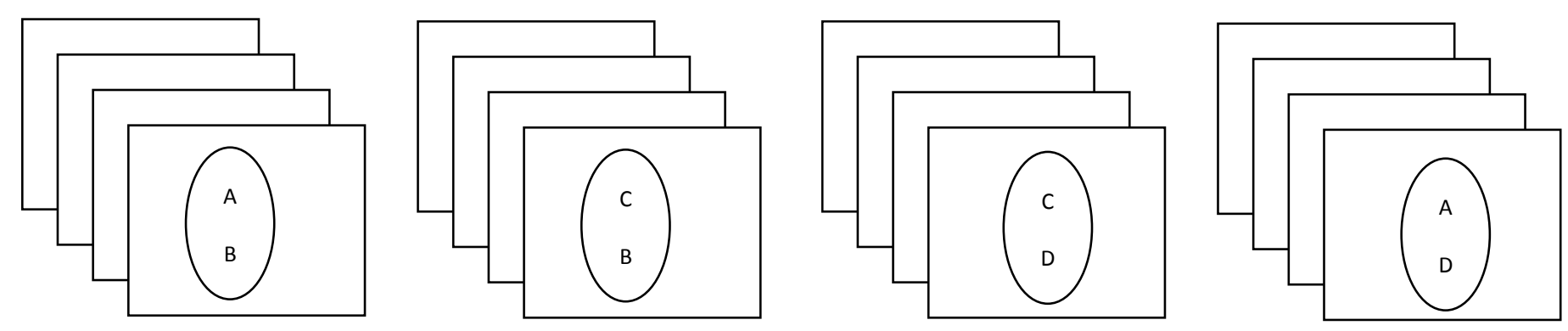


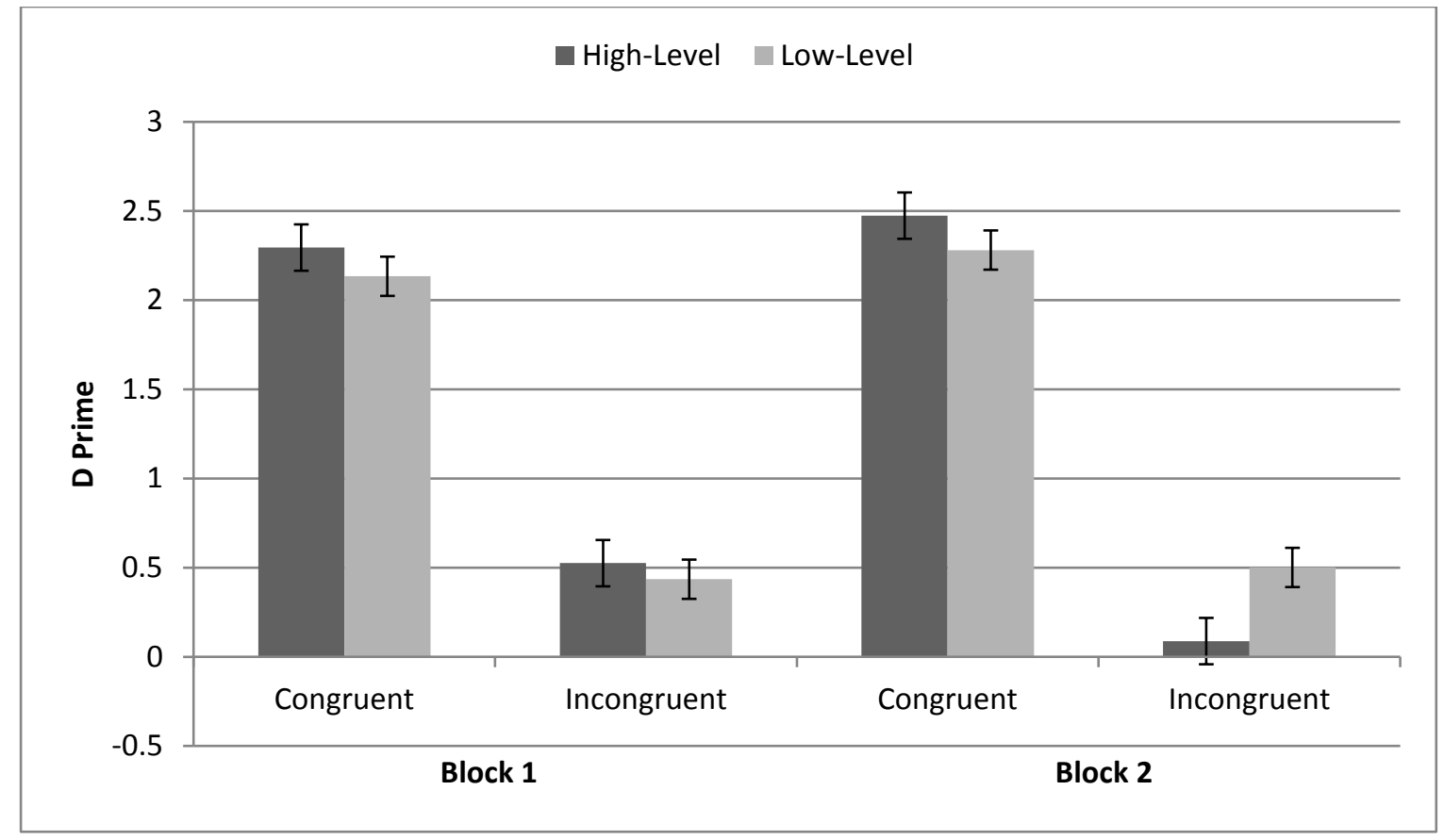

